\title{
Trastornos sensitivos genitales y maltrato psicológico en la relación de pareja. Una revisión bibliográfica
}

\section{Sensitive genital disorders and psychological abuse in the couple relationship. A bibliographic review}

\author{
Camila Gutiérrez-Basso ${ }^{\text {* }}$, María C. Pantoja-Robles², María E. Herane ${ }^{2}$, Magdalena Nalda², \\ Carlos Wenzel2, Jorge Lecannelier², Daniella Espínola-Marín'2,3 y Rodrigo Guzmán-Rojas²
}

${ }^{1}$ Departamento de Psicología Clínica, Psiconsulta.cl; ${ }^{2}$ Departamento de Ginecología y Obstetricia, Unidad de Piso pélvico, Facultad de Medicina, Clínica Alemana, Universidad del Desarrollo; ${ }^{3}$ Departamento de Cirugía, Hospital Padre Hurtado. Santiago, Chile

\section{Resumen}

Introducción: Los trastornos sensitivos genitales y su relación con el maltrato psicológico en la pareja es un tema poco tratado en la literatura y, sin embargo, presente en la entrevista médica y psicológica. Aplica para aquellos casos en que no se aprecia alteración en la exploración física suficiente como para atribuirlo a causas orgánicas. Objetivo: Recopilar e integrar diferentes visiones acerca del síntoma de insensibilidad genital durante las relaciones sexuales, definir si este síntoma corresponde a una disfunción sexual e indagar sobre su relación con la violencia psicológica en la relación de pareja. Método: Revisión bibliográfica. Resultados: Los trastornos sensitivos cuya base orgánica es descartada no corresponderían a una disfunción sexual, sino más bien a una alteración sensorial disociativa como respuesta a una situación de violencia psicológica acompañada de violencia sexual en todos los casos. Conclusiones: La insensibilidad genital es un tema que debe ser considerado al hacer la anamnesis de una paciente, para así ser derivada a un especialista con la intención de abordarlo de forma interdisciplinaria y, por ende, evaluar y diferenciar los elementos orgánicos y funcionales del síntoma.

Palabras clave: Sexualidad. Maltrato psicológico. Violencia en la pareja. Violencia sexual. Trastornos sensitivos genitales. Alteraciones sensoriales disociativas.

\section{Abstract}

Introduction: Genital sensory disorders and how they relate to psychological abuse in the couple is a topic that is scarcely address in the literature, however present it might be in medical and psychological interviews. It is often found in cases where there is no perceived sufficient alteration in physical examinations in order to attribute it to organic causes. Objective: To collect and integrate different views regarding the symptom of genital insensitivity during sexual intercourse, define whether this symptom relates to sexual dysfunction and inquire about its connection with psychological violence in the couple. Method: Bibliographic assessment. Results: Sensory disorders, whose organic basis is ruled out, do not relate to sexual dysfunction but rather to a dissociative sensory alteration in response to a situation of psychological violence accompanied, in all cases, by sexual violence. Conclusions: Genital insensitivity is an issue that must be taken into account at the time of taking the patient's medical history. This way, it can be referred to a specialist with the intention of addressing it in an interdisciplinary way and, therefore, evaluate and differentiate the organic and functional elements of the symptom.

Key words: Sexuality. Psychological abuse. Intimate partner violence. Sexual violence. Genital sensory disorders. Dissociative sensory disorders.

Correspondencia:

${ }^{*}$ Camila Gutiérrez-Basso

Fecha de recepción: 17-10-2020

Fecha de aceptación: 15-10-2021

DOI: 10.24875/RECHOG.M21000033 E-mail: cgbasso@gmail.com
Disponible en internet: 23-12-2021 Rev Chil Obstet Ginecol. 2021;86(5):497-501 www. rechog.com 0048-766X / @ 2021 Sociedad Chilena de Obstetricia y Ginecología. Publicado por Permanyer. Éste es un artículo open access bajo la licencia CC BY-NC-ND (https://creativecommons.org/licenses/by-nc-nd/4.0/). 


\section{Introducción}

Esta revisión bibliográfica nace del interés del equipo multidisciplinario para saber más sobre aquellas pacientes que señalan tener una disminución en la sensibilidad de sus genitales durante las relaciones sexuales. Para lo anterior, el punto de partida es definir si se trata de una disfunción sexual y si tiene relación con la violencia psicológica conyugal. El «no sentir» es un motivo de consulta que aparece solo durante una entrevista más exhaustiva en consulta ginecológica. Debido a ello, se trata de un tema poco estudiado. Esto podría atribuirse a que la insensibilidad en las relaciones sexuales no genera dolor ni placer. Por lo tanto, este síntoma puede parecer poco relevante para la paciente, o incluso normal, y además podría tener un carácter adaptativo y funcional importante. La violencia psicológica en la pareja implica a ambos sexos; igualmente existen hombres víctimas de esta realidad, pero en esta revisión nos centramos en el síntoma femenino.

\section{Método}

Tratándose de un tema poco abordado en la literatura científica durante la última década, se realizó una revisión de artículos de revistas científicas y literatura entre los años 1989 y 2017, con los términos utilizados en el título o resumen. Tanto en Scielo como en Web Of Science se buscaron artículos utilizando las palabras clave "Genital sensory disorders", "Dissociative sensory disorders" e "Insensibilidad genital", sin resultados. Por lo anterior, se buscó información en sitios de universidades, tesis de doctorado y libros relacionados en inglés, español y francés.

\section{Desarrollo}

El primer paso es indagar si se trata de una disfunción sexual. El concepto de insensibilidad no está recogido en los manuales diagnósticos actuales: «frigidez» es el concepto más cercano a lo que se desea apuntar en esta revisión, entendida como la carencia de respuesta sexual femenina de forma generalizada. Aparecen también el «trastorno del deseo»" y la "disfunción orgásmica femenina», definida como la dificultad de grado variable para experimentar el orgasmo. Sin embargo, estos últimos apuntan a otras fases específicas de la relación sexual, donde la mujer puede sentir y obtener placer con su pareja, ya sea sin orgasmo o en un inicio con falta de deseo. Dentro de las disfunciones sexuales encontradas en la revisión, ninguna responde específicamente al síntoma estudiado.

Podría tratarse de una alteración en la propiocepción en la que el déficit en la sensibilidad lleva a ausencia de placer físico y emocional. Cuando este «adormecimiento" se manifiesta de forma secundaria, habiendo tenido previamente sensibilidad, esto puede sugerir un carácter situacional ${ }^{\dagger}$. Este último punto es un tema preponderante en esta revisión. Lo anterior debido a que las pacientes, tarde o temprano, manifestarán encontrarse en una situación de conflicto en la relación de pareja.

Cuando las pacientes comienzan a describir las situaciones que viven en el ámbito de pareja, su motivo de consulta se complejiza. Estos casos se presentan frecuentemente ligados a situaciones de violencia psicológica conyugal; un patrón regular de conductas y actitudes violentas o coercitivas por parte de sus parejas, centradas en conseguir la sumisión y el control en la relación. Se trata de una violencia invisible, puesto que, al no tratarse de lesiones físicas, no deja huellas visibles en la persona ${ }^{2}$.

Según la Organización Mundial de la Salud (1998), el aspecto más dañino del maltrato no es la violencia en sí misma, sino vivir con miedo y terror. Este aspecto está muy presente en el relato de las pacientes. Deja una huella que va haciendo visibles trastornos psicopatológicos que requieren atención médica y psicológica? ${ }^{2}$.

Los trastornos de la esfera sexual se encuentran con mayor frecuencia en el contexto de una relación de pareja violenta en curso, como consecuencia directa del desequilibrio y la disfuncionalidad ${ }^{3}$. En una revisión de 2007, Cocker $^{+}$evidencia la fuerte asociación entre los trastornos de la esfera sexual y la violencia intrafamiliar, presente en 17 de los 18 estudios considerados. Estos fueron principalmente vulvodinia, dispareunia y cistitis intersticial. Además, ser o haber sido víctima de violencia a menudo conduce a comportamientos sexuales de riesgo, como la promiscuidad y la prostitución. En contraste, también puede darse una reducción del deseo sexual y problemas para alcanzar el orgasmo.

Es posible encontrar literatura referente al adormecimiento corporal como respuesta a un estresor, pero pocos autores se refieren específicamente a los trastornos de sensibilidad genital femenina. La psicoanalista Maryse Choisy ${ }^{5}$ habla acerca de la relación entre la insensibilidad y la violencia contra la mujer. En su estudio de la prostitución desde un enfoque médico 
psicológico y social, describe «una suerte de adormecimiento de las zonas genitales que prepara a la mujer para entregarse a cualquier hombre sin sentir, donde las tendencias a la autodestrucción convergen. Aquí se produce la unión entre un hombre y una mujer que se aborrecen». La autora define la insensibilidad como un mecanismo de defensa que permite al cuerpo desentenderse, alejarse de ese escenario. Como las prostitutas relatan: "yo no estaba allí, era solo mi cuerpo". Este estado únicamente es soportado a costa de imponer a la mente un apartamiento de la conciencia. En relación a lo anterior, esto correspondería a los actuales criterios de despersonalización y desrealización característicos de un trastorno disociativo. En estas pacientes se aprecia cómo el individuo se siente escindido de su propio cuerpo ${ }^{6}$.

En 2002, Judith Trinquart, médica forense de la universidad Paris XIII, desarrolló este tema en su tesis de doctorado: el hecho de tener relaciones sexuales repetitivas sin consentimiento en sí es una agresión. Esto genera una disociación psíquica que deriva en trastornos sensitivos del esquema corporal: hipoestesia que con el tiempo evoluciona a anestesia. Esto eleva el umbral del dolor, haciendo a las pacientes más tolerantes o insensibles; a tal punto, que se enteran tarde de las patologías ginecológicas durante los controles habituales. Destaca la importancia de ver este tema más allá del abordaje médico, en sus ámbitos relacional, social, psicológico y humano, es decir, de manera general.

Los trastornos disociativos mencionados por las autoras anteriores están descritos actualmente en el DSM-V ${ }^{6}$ y se caracterizan por una interrupción o una discontinuidad en la integración normal. Abarcan múltiples áreas, entre ellas la percepción y el comportamiento. Mascayano et al. $^{8}$ describen un subtipo particular de disociación (trastorno disociativo no especificado en el DSM-IV y otros trastornos en CIE-10 ${ }^{\circ}$ ) definido como «anestesias y pérdidas sensoriales disociativas»: aparición de alteraciones exclusivamente de tipo sensitivo-sensorial que no son consecuencia de lesiones neurológicas. Las más frecuentes son las pérdidas o alteraciones de la sensibilidad cutánea y las pérdidas visuales, manteniéndose intactas la movilidad y las funciones motrices. Puede presentarse, además, parestesia. Considerando lo anterior, los trastornos de la sensibilidad no serían una disfunción sexual propiamente tal. En consecuencia, la revisión fue llevando el tema hacia la rama de la psiquiatría, demostrando la importancia de un abordaje multidisciplinario.
Considerando los aportes de los autores mencionados, debemos interrogarnos sobre la relación entre los estudios acerca de las prostitutas y el trastorno sensitivo genital en las mujeres que llevan una relación de pareja convencional, alejadas de esa realidad. Aquí, si bien son contextos diferentes, el factor común es la violencia sexual ${ }^{10}$ : acceden reiteradas veces a tener relaciones sexuales no deseadas, de manera sostenida en el tiempo.

La mujer que es forzada constantemente a tener relaciones sexuales, sin utilizar ninguna acción física de por medio, sino amenazas implícitas, sin la más mínima contrapartida afectiva, ignorando incluso una discusión reciente, es víctima de violencia sexual. El ejercicio de esta violencia sostenida en el tiempo produciría indefensión aprendida. Lo anterior desestructura la psique hasta llevarla a un estado disociativo, con múltiples alteraciones psicosomáticas y dependencia afectiva y emocional con su victimario ${ }^{11}$. Adicionalmente, Amor y Echeburúa ${ }^{12}$ describen la dependencia emocional hacia el agresor como parte del maltrato psicológico: una vivencia de emociones contradictorias, vínculo emocional basado entre la intermitencia del buen y el mal trato, enamoramiento intenso y miedo al abandono. Algunos autores postulan que el perfil del maltratador obedece a una línea de psicopatía integrada (subclínica). Son personas que parecen tener en abundancia los rasgos más deseados por las personas normales: son encantadores, inteligentes y exitosos con el sexo opuesto. Sin embargo, estos son atributos superficiales, puesto que son incapaces de proporcionar una relación íntima de respeto y amor, siendo más frecuente su recurso a la violencia psicológica que a la física ${ }^{13}$. Existen discrepancias entre el comportamiento que muestran en público y el que mantienen en privado, lo que genera confusión y aislamiento en la víctima ${ }^{14}$. Lo visto demuestra que existen perfiles psicológicos de mujeres más propensas a sufrir el síntoma estudiado: aquellas que presentan rasgos de dependencia emocional podrían llegar a ser víctimas de una relación abusiva, y desarrollar indefensión aprendida y su consecuente estado disociativo.

Muchas víctimas de maltrato psicológico y sexual no son conscientes de que lo son. Atribuyen los sucesos al carácter de su pareja o intentan minimizar su trascendencia, por lo que es necesario abordar el tema con tacto y empatía ${ }^{2}$. Existe en algunas mujeres maltratadas el concepto de amor romántico que implica depender del otro y adaptarse a él, postergando incluso lo propio $^{15}$. 
Uno de los factores socioculturales que podría favorecer la normalización de esta disociación cuerpo-mente, en algunos grupos de pacientes, es la influencia de la formación espiritual y religiosa, la cual en muchas sociedades determina la arquitectura de la sexualidad de sus fieles. Algunas religiones, como la judía, la musulmana y la hindú, conciben la sexualidad como un medio tanto para mantener la especie como para la obtención de placer. Por el contrario, la religión católica tradicionalmente relegó al sexo solo para fines reproductivos, castigando el disfrute de la sexualidad, incluso en el contexto matrimonial ${ }^{16}$. Sin embargo, es justo señalar que el Concilio Vaticano II (1962-1965) aceptó el placer sexual en el contexto matrimonial como aporte a la estabilidad de la pareja. La literatura es escasa al abordar la relación entre la sexualidad y la religión en heterosexuales, y más aún en mujeres. En 1989, Pepe et al. ${ }^{17}$ publicaron un estudio prospectivo de 360 mujeres católicas, 144 observantes y 216 no observantes. Este mostró que las católicas observantes tienen relaciones sexuales menos satisfactorias ( $44 \%$ vs. $74 \%$ ), han sido más solicitadas por sus parejas para cambiar su forma de tener sexo ( $57 \%$ vs. $28 \%$ ), y rechazan en mayor proporción la solicitud del profesional de la salud en entregar información sobre su vida sexual $(24 \%$ vs. $0,1 \%)$ que las mujeres católicas no observantes. En suma, la influencia y la intensidad de las creencias religiosas podrían ser un factor normalizador de la disociación de la mujer que estaría involucrada en una relación de pareja disfuncional.

Como último punto, es necesario referirse al papel de los ginecólogos en la búsqueda y el diagnóstico de esta condición. Son los ginecólogos quienes tienen acceso periódico (en muchos casos anualmente) a sus pacientes. Cuentan, además, con la confianza de estas, y su examen clínico y físico está directamente relacionado con los órganos que pueden verse comprometidos en su vida sexual. Cabe preguntarse, por lo tanto, si la formación en sexualidad de los ginecólogos es adecuada. En los posgrados de ginecología y obstetricia, la formación en sexualidad es hasta el día de hoy deficiente. En 2015, Vieira et al. ${ }^{18}$ publicaron que un $63 \%$ de los becados de ginecología en Brasil no tenía formación alguna en sexualidad, y un $29 \%$ tuvo menos de 6 horas de formación en ese tema. El 56\% no hacía la historia sexual de sus pacientes, el 51\% no se sentía preparado para responder preguntas sobre sexualidad y el $84 \%$ atribuía estas dificultades a la falta de formación obtenida.

Un 76\% de ginecólogos de Gran Bretaña consideran su formación en sexualidad deficiente ${ }^{19}$. El mismo estudio señala que un $70 \%$ de los ginecólogos no evalúan la sexualidad de sus pacientes por falta de tiempo en la consulta, un $25 \%$ porque no están seguros de las opciones terapéuticas disponibles y otro $20 \%$ porque no saben qué o cómo preguntar (más de una respuesta por profesional). Por lo tanto, una paciente con esta condición, que habitualmente no reconoce que tiene un problema, al ser evaluada por un profesional que no lo busca de manera dirigida o que desconoce su existencia, está condenada a mantener su estado indefinidamente. Considerando lo anterior, sería beneficioso profundizar el tema de la sexualidad y la relación de pareja en la formación de los ginecólogos con el fin de pesquisar mejor estos fenómenos.

\section{Tratamiento}

Una mujer víctima de maltrato psicológico y sexual experimentará altos niveles de temor al pensar que su relación pueda terminar. Sin embargo, es una persona que podría salir del círculo vicioso de la violencia en la pareja al comprender su situación desde lo racional y emocional, con la ayuda de un equipo multidisciplinario. Las intervenciones psicológicas deben contemplar la dimensión de la dependencia emocional para evitar perpetuar relaciones de maltrato ${ }^{15}$. Una vez realizado este trabajo de reparación, la mujer debe aprender a sensibilizarse con respecto a las sensaciones que ha reprimido defensivamente durante largo tiempo. Para conseguir una relación que facilite este proceso, suele exigirse una terapia de pareja previa a cualquier intervención de terapia sexual. El enfoque de estas terapias es de tipo integrativo, con una mayor influencia de la corriente cognitiva conductual ${ }^{1}$. En algunos casos se puede complementar con terapia kinésica del piso pélvico. Weiss et al. ${ }^{20}$ reportaron que el entrenamiento regular del piso pélvico en la clínica y en casa mejora su función de soporte, incrementa el flujo sanguíneo y estimula la propiocepción de la zona, contribuyendo a un orgasmo más intenso. En un estudio realizado con 236 mujeres en la Universidad Piloto de Colombia ${ }^{21}$ se logró comprobar que el nivel de significancia entre las variables de maltrato y resiliencia es positivo, es decir, que la experiencia de estas mujeres en sus relaciones de pareja y con historia de maltrato las ha llevado a desarrollar un aprendizaje positivo, volviéndose resilientes, o sea, que buscan nuevas posibilidades donde no se presente el maltrato. Esto refuerza que toda propuesta de tratamiento es prometedora, incentiva la capacidad de superación en la persona y ayuda a eliminar el estigma de «víctima irreversible» en la mujer. 


\section{Conclusiones}

Los trastornos sensitivos genitales no serían una disfunción sexual, sino un trastorno disociativo de carácter situacional. Este responde a una situación de violencia psicológica y sexual en la pareja. Existe una propensión mayor de estos trastornos cuando hay violencia psicológica y sexual en la pareja. Los trastornos sensitivos genitales no afectan la frecuencia de las relaciones sexuales. Al contrario, es una conducta que intenta evitar conflictos con el cónyuge y, por ende, lleva a tener relaciones sexuales sin consentimiento de manera sostenida en el tiempo. En esta revisión aparece un concepto específico de maltrato que debe acompañar a la violencia psicológica: la violencia sexual. De ahí su génesis: parte de una mujer que no logra poner límites a diversas agresiones y desea al mismo tiempo cumplir las expectativas del otro por temor al abandono. El cuerpo se disocia, activando mecanismos de defensa (despersonalización) para poder sobrellevar una situación difícil de integrar y enfrentar. Si se suman a esta condición un medio sociocultural que castiga el goce de la sexualidad y un cuerpo médico que no busca activamente las sintomatologías asociadas (por desconocimiento o por falta de herramientas), tenemos como resultado un grupo de mujeres que difícilmente serán tratadas. Por el contrario, si se aborda en el marco de un equipo multidisciplinario, este puede significar una red de apoyo que incentive la tendencia resiliente en la mujer maltratada. Permitiría que esta se abra a nuevas posibilidades de relaciones de pareja sanas, centradas en el afecto y el respeto recíproco en la pareja.

El grupo de autores considera relevante esta revisión bibliográfica. Ha despertado interés en poder realizar a futuro una investigación de enfoque cuantitativo para medir estos factores y comprobar su relación. En consecuencia, permitiría establecer vías clínicas y protocolos de tratamiento multidisciplinario. De ser así, la disminución en la sensibilidad genital durante las relaciones sexuales podría ser un problema tratable y reversible.

\section{Financiamiento}

Este manuscrito no contó con financiamiento, todos colaboramos de forma voluntaria.

\section{Conflicto de intereses}

Los autores declaran no tener ningún conflicto de intereses.

\section{Responsabilidades éticas}

Protección de personas y animales. Los autores declaran que para esta investigación no se han realizado experimentos en seres humanos ni en animales.

Confidencialidad de los datos. Los autores declaran que han seguido los protocolos de su centro de trabajo sobre la publicación de datos de pacientes.

\section{Derecho a la privacidad y consentimiento infor-}

mado. Los autores declaran que en este artículo no aparecen datos de pacientes.

\section{Bibliografía}

1. KAPLAN H. Manual ilustrado de terapia sexual. La solución a los trastornos sexuales más comunes. Barcelona: Debolsillo; 2010.

2. Hernández Ramos $C$, Magro Servet V, Cuéllar Otón JP. El maltrato psicológico. Causas, consecuencias y criterios jurisprudenciales. El problema probatorio Aequitas. 2014;3(7):27-53.

3. Costantini E, Villari D, Filocamo M. Sexuality after abuse. En: Costantini E, Villari D, Filocamo M, editores. Female sexual function and dysfunction. Switzerland: Springer International Publishing; 2017. p. 123-34.

4. Cocker AL. Does physical intimate partner violence affect sexual health? A systematic review. Criminol Penol. 2007;5:55.

5. Choisy M. Prostitución: enfoque médico-psicológico y social. Buenos Aires: Lumen-Horme; 1993.

6. López CA. Manual diagnóstico y estadístico de los trastornos mentales: DSM-5. Madrid: Médica Panamericana; 2014.

7. Trinquart J. La décorporalisation dans la pratique prostitutionnelle: un obstacle majeur à l'accès aux soins. Médecine générale Bobigny Thèse de doctorat; 2002.

8. Mascayano F, Maray F, Roa A. Trastornos disociativos: una pérdida de la integración. Psiquiatría Universitaria. 2009;5(3):379-88.

9. López-lbor J. CIE-10: Trastornos mentales y del comportamiento. Madrid: Meditor; 1992.

10. Cáceres Carrasco J. Violencia física, psicológica y sexual en el ámbito de la pareja: papel del contexto. Clínica y Salud. 2004;15:33-54.

11. Pérez LFA. La prueba pericial psicológica en asuntos de violencia de género. Revista Internauta de Práctica Jurídica. 2008;21:15-29.

12. Amor PJ, Echeburúa $E$. Claves psicosociales para la permanencia de la víctima en una relación de maltrato. Clínica. 2010;1:97-104.

13. Pozueco J, Moreno J, Blázquez M, García-Baamonde M. Psicópatas integrados/subclínicos en las relaciones de pareja: perfil, maltrato psicológico y factores de riesgo. Papeles del Psicólogo. 2013;34:32-48.

14. Alonso MB, Manso JMM, Sánchez MEG-B. Revisión teórica del maltrato psicológico en la violencia conyugal. Psicología y Salud. 2010;20:65-75.

15. Aiquipa Tello JJ. Dependencia emocional en mujeres víctimas de violencia de pareja. Revista de Psicología (PUCP). 2015;33:411-37.

16. Kellogg Spadt S, Rosenbaum TY, Dweck A, Millheiser L, Pillai-Friedman S, Krychman M. Sexual health and religion: a primer for the sexual health clinician (CME). J Sex Med. 2014;11:1607-18.

17. Pepe F, Panella M, Pepe G, D'agosta S, Pepe P. Frequency of sexual dysfunctions among Roman Catholic women. Fam Pract. 1989;6:16-8.

18. Vieira TC, de Souza E, da Silva I, Torloni MR, Ribeiro MC, Nakamura MU. Dealing with female sexuality: training, attitude, and practice of obstetrics and gynecology residents from a developing country. J Sex Med. 2015;12:1154-7.

19. Roos AM, Thakar R, Sultan AH, Scheer I. Female sexual dysfunction: are urogynecologists ready for it? Int Urogynecol J Pelvic Floor Dysfunct. 2009;20:89-101.

20. Weiss PM, Rich J, Swisher E. Pelvic floor spasm: the missing link in chronic pelvic pain. Published on Contemporary OB/GYN (http://contemporaryobgyn.modernmedicine.com). January 2012Contemporary Ob/gyn 57(10):38-46.

21. Roa C, Estrada K, Tobo M. Nivel de resiliencia en mujeres maltratadas por su pareja. Revista Con-textos. 2012;(8). Universidad Piloto de Colombia. Disponible en: http://repository.unipiloto.edu.co/handle/20.500.12277/9041 